\title{
Solidificación / Estabilización de residuos orgánicos mediante granulación con sepiolita
}

\author{
F. A. LÓPEZ Y A. LÓPEZ-DELGADO \\ Dpto. de Reciclado de Materiales y Residuos. Centro Nacional de Investigaciones Metalúrgicas. Consejo Superior de \\ Investigaciones Científicas (CSIC). Avda. Gregorio del Amo,8. 28040 Madrid.
}

\begin{abstract}
Se estudia un proceso de solidificación/estabilización con sepiolita, de diversos tipos de residuos industriales conteniendo contaminantes orgánicos considerados tóxicos y peligrosos (pinturas, cosméticos, tensioactivos, resinas...). El proceso se realiza mezclando el residuo y la arcilla hasta obtener un material granulado, de baja humedad y punto de inflamación próximo a $100^{\circ} \mathrm{C}$. El tratamiento individualizado con sepiolita de cada uno de los residuos estudiados, indica que puede obtenerse un material adecuado para su almacenamiento en un depósito de residuos, o bien, un combustible de residuo (CDR) apto para su revalorización energética en hornos de fabricación de clinker. En el caso de un tratamiento conjunto de todos los residuos con la arcilla, se obtiene un material cuyo destino más adecuado sería su revalorización energética. La cantidad de arcilla utilizada varía según el tipo de residuo y está comprendida entre 0.3 y $0.6 \mathrm{~kg} / \mathrm{kg}$ de inerte final. Se estudian y caracterizan químicamente cada uno de los materiales obtenidos. Finalmente, algunos de ellos se estudian mediante mediante microscopía de barrido a $-190{ }^{\circ} \mathrm{C}$.
\end{abstract}

Palabras Clave: Sepiolita; Residuos Orgánicos; Solidificación; Estabilización Granulación

Solidification / Stabilization of industrial organic wastes by granulation with sepiolite

A solidification/stabilization process of different toxic and hazardous organic industrial wastes with sepiolite was studied. Wastes coming from, among other, paint, cosmetic, resin, liquid detergent industries were mixed with the clay. The process yielded granulated solids with low moistness and ignition point close to $100^{\circ} \mathrm{C}$. The materials so obtained can be stored into a waste deposit or used as a combustible to cement klin. This last option involves an energy recovery and accordingly allows the complete utilisation of a waste's calorific value. Clay/inert product mass rates ranged between 0.3-0.6, depending on the type of waste. Chemical characterisations of final materials were performed and crio-scanning electron microscopy (at $-190^{\circ} \mathrm{C}$ ) were employed to study the sorption process.

Keywords: Sepiolite; Industrial Organic Waste; Solidification; stabilization; Granulation.

\section{1.- INTRODUCCIÓN}

El Catálogo Europeo de Residuos clasifica a los residuos orgánicos dentro de los residuos especiales, tóxicos y peligrosos, agrupándolos en las siguientes categorías: disolventes y residuos líquidos disolventes; residuos aceitosos (aceites, grasas e hidrocarburos); productos químicos y fitosanitarios; residuos líquidos orgánicos; pinturas, tintas y colorantes y productos caducados y restos de fabricación de orgánicos. A nivel del Estado español, no se conocen datos concretos sobre su producción, pero se estima en 1.500 .000 t/año.

$\mathrm{Al}$ tratarse de residuos especiales, su gestión actual es, por una parte, su almacenamiento en depósitos de seguridad o su destrucción térmica en plantas de incineración de residuos industriales. El almacenamiento en depósitos de seguridad es un modo de gestión que va a experimentar importantes modificaciones en los próximos años coincidiendo con la entrada en vigor de la Directiva Comunitaria $\operatorname{COM(97)~} 105$ final 97/008 (SYN). De acuerdo a esta Normativa, los residuos orgánicos deberán ser sometidos a un tratamiento previo de inertización o estabilización como condición imprescindible para su depósito.
El empleo de arcillas tanto naturales como modificadas térmica o químicamente, sobre todo esmectitas, caolinitas y sepiolita, en la eliminación de contaminantes orgánicos en solución acuosa, ha sido ampliamente descrita en la literatura. En especial, en lo que se refiere a su utilización a nivel de laboratorio en la eliminación de aminas, pesticidas, herbicidas y compuestos orgánicos diversos $(1-5)$.

Respecto a los procesos de estabilización/solidificación de residuos orgánicos la utilización de cementos, tradicionalmente empleados para residuos inorgánicos, está limitada como consecuencia de los efectos negativos que los compuestos orgánicos producen en los procesos de hidratación del cemento (6).

Para paliar este problema se han desarrollado procedimientos alternativos consistentes en tratar previamente al residuo, normalmente con cal, para su adecuación al proceso de inertización con cemento (7). Así mismo, se ha estudiado la inertización de residuos mediante el empleo conjunto de arcillas y cemento, sobre todo para la fijación de contaminantes inorgánicos conteniendo metales pesados (8) o compuestos 
orgánicos (9) así como la utilización de arcillas organofílicas y arcillas modificadas con complejos orgánicos para la estabilización de contaminantes orgánicos, normalmente presentes en soluciones acuosas u orgánicas $(10,11)$.

El objetivo de estos tratamientos es la obtención de sólidos monolíticos de resistencia elevada, ya sea a la compresión (resistencia mecánica) y sobre todo a la lixiviación de los contaminantes (resistencia química).

En este trabajo se describe un procedimiento para la solidificación y estabilización físico-química de residuos orgánicos procedentes de diversos procesos industriales (12). El proceso se basa en el empleo, bajo determinadas condiciones, de un filosilicato (sepiolita) de elevada superficie específica que permite obtener materiales inertes.

Las diferencias fundamentales frente a otros procedimientos de estabilización son, entre otras, las siguientes:

a) No empleo de cemento y en consecuencia, ausencia de agua y fraguado.

b) Posibilidad de tratamiento de cualquier tipo de residuo orgánico, ya sea líquido o pastoso.

c) Obtención de un material final que puede ser revalorizado energéticamente (combustible de residuo (CDR)).

\section{2.- EXPERIMENTAL}

\section{1.- Residuos tratados}

Se han estudiado 15 muestras diferentes de residuos procedentes de diversos procesos industriales: residuos producidos en la fabricación de detergentes líquidos (tensioactivos); residuos procedentes de la fabricación de pinturas; materias primas caducadas de la fabricación de cosméticos; lodos resultantes de las operaciones de limpiezas de tanques que contuvieron hidrocarburos clorados; tintas y finalmente restos de pinturas.

Complementariamente, se estudió el comportamiento de una mezcla de los residuos anteriores. La mezcla se considera una muestra media de los residuos orgánicos que se tratan en un Centro de Tranferencia y Tratamiento Físico-químico. Todas las muestras fueron suministradas por la empresa Cartera Ambiental, S.A (Ajalvir.Madrid).

En la Tabla 1 se recogen las principales características físico-químicas de los residuos estudiados.

\section{2.- Arcilla empleada}

Se utilizó una sepiolita suministrada por Tolsa S.A (Vicálvaro, Madrid). Su composición química, se determinó mediante análisis químico realizado por absorción atómica sobre una solución obtenida a partir de la fusión de una muestra de arcilla con tetraborato de litio en crisol de platino y posterior disolución en $\mathrm{HCl}$ diluido. El contenido en sílice se determinó mediante gravimetría y las pérdidas por calcinación a $1000^{\circ} \mathrm{C}$ se obtuvieron mediante análisis termogravimétrico utilizando una termobalanza Shimadzu TA $50 \mathrm{H}$ (atmósfera de aire de $20 \mathrm{ml} / \mathrm{min}$; velocidad de calentamiento de $20^{\circ} \mathrm{C} / \mathrm{min}$ y crisol de alúmina y referencia interna de $\left.\alpha \mathrm{Al}_{2} \mathrm{O}_{3}\right)$.

La composición mineralógica se determinó mediante difracción de RX utilizando un difractómetro Siemens D-501 con radiación monocromática $\mathrm{CuK} \alpha$. La fracción de la arcilla se determinó realizando un agregado orientado en etilenglicol que se estudió mediante difracción de RX. La superficie específica BET se determinó mediante adsorción de nitrógeno a 77K utilizando un equipo Coulter SA 3100. La muestra se desgasificó a $180^{\circ} \mathrm{C}$ durante $180 \mathrm{~min}$.

TABLA 1.- CARACTERÍSTICAS FÍSICO-QUÍMICAS DE LOS RESIDUOS ESTUDIADOS

\begin{tabular}{|c|c|c|c|c|c|c|}
\hline Residuo & $\begin{array}{c}\text { Lodos } \\
\text { Limpieza }\end{array}$ & Cosméticos & Resinas & Pinturas & Tensioactivos & $\begin{array}{r}\text { Mezcla } \\
\text { Media }\end{array}$ \\
\hline Densidad (g.cm ${ }^{-3}$ ) & $1.22-1.25$ & $0.82-0.88$ & 1.50 & $0.98-1.26$ & 1.40 & 1.15 \\
\hline Viscosidad (cPs) & n.d & n.d & n.d & $7510-7650$ & 380 & n.d \\
\hline Punto Inflamación $\left({ }^{\circ} \mathrm{C}\right)$ & $30-47$ & $52-67$ & $<30$ & $<30-35$ & 78 & $<\mathbf{3 0}$ \\
\hline PCS (kcal/kg) & $1000-5667$ & 2909-8516 & 9473 & 4744-8077 & $<1000$ & 5450 \\
\hline $\operatorname{PxC} 105^{\circ} \mathrm{C}(\%)$ & 27.1-29.9 & $58.6-83.2$ & 50.1 & $31.6-80.2$ & 45.1 & 45.1 \\
\hline Cloro Total (\%) & $0.37-19.29$ & $0.15-0.38$ & 0.12 & $0.1-2.69$ & 20.50 & 2.40 \\
\hline Azufre Total (mg/kg) & n.d & n.d & n.d & n.d & n.d & 1106 \\
\hline Volátiles (\%) & n.d & n.d & n.d & n.d & n.d & 37.8 \\
\hline Metales Totales $(\mathrm{mg} / \mathrm{kg})$ & $340-923$ & $1565-11000$ & 535 & 417-9671 & 413 & 7360 \\
\hline Humedad (\%) & $<1$ & $1.8-56$ & 0.22 & $0.2-8-4$ & 49.60 & 7.3 \\
\hline PH & $5.5-9.8$ & 6-9 & ---- & $3.5-8.0$ & $<1$ & 5.6 \\
\hline
\end{tabular}

n.d. $=$ no determinado

(Los valores de los intervalos, corresponden a los obtenidos para diferentes residuos estudiados dentro de un mismo grupo). 


\section{3.- Proceso de solidificacion/estabilizacion}

El procedimiento utilizado consiste en realizar una mezcla del residuo con sepiolita (12). Las mezclas se realizaron en una mezcladora horizontal de laboratorio MAP modelo MLH 06 de 5 litros de capacidad de carga. La velocidad del rotor de mezcla, de tipo tajadera, fue de $300 \mathrm{rpm}$. Los tiempos de mezcla se variaron entre 10 y $15 \mathrm{~min}$. Como resultado se obtuvieron unos sólidos granulados, con diferentes tamaños de granos, dependiendo de las proporciones de las mezclas y del tipo de residuo. Se realizaron diversas ensayos variando las proporciones relativas de arcilla y residuo en el intervalo comprendido entre 0.4 y $0.6 \mathrm{~kg}$ de arcilla por $\mathrm{kg}$ de mezcla. Para mezclas con contenidos inferiores a 0.4 y superiores a 0.6 $\mathrm{kg} / \mathrm{kg}$ no se obtuvieron resultados satisfactorios.

En el caso de los residuos procedentes de la fabricación de pinturas, las muestras se trataron previamente en un rotavapor con el fin de recuperar los disolventes orgánicos que contienen. Los residuos sólidos obtenidos de esta operación de destilación se sometieron al proceso de solidificación/estabilizaron tal y como se ha descrito anteriormente.

\section{4.- Análisis y control de los materiales finales}

Una vez realizadas las mezclas, se determinó la toxicidad de los materiales obtenidos siguiendo el protocolo TCLP de la EPA (13), consistente en la lixiviación de los sólidos con ácido acético $4 \mathrm{~N}$ durante 24 horas. Transcurrido este tiempo los sólidos se separaron de los lixiviados mediante filtración, analizándose en estos últimos el contenido en metales, la demanda química de oxígeno, DQO y la ecotoxidad.

El contenido en metales se determinó mediante absorción atómica utilizando un equipo Philips Pye Unicam SP9. La DQO fue obtenida mediante el método normalizado de digestión con $\mathrm{K}_{2} \mathrm{Cr}_{2} \mathrm{O}_{7}$ utilizándose un colorímetro Hach modelo DR800.

Para la determinación de la ecotoxicidad se empleó el método de bioluminiscencia utilizando photobacterium phosphoreum en un equipo Microtox M500. El valor del EC50 (concentración efectiva del lixiviado que disminuye la emisión de luz de las bacterias en un $50 \%$ ) se midió a $15^{\circ} \mathrm{C}$ después de 15min. de incubación (14).

El resto de los análisis químicos, cuya descripción se omite, se llevó a cabo siguiendo métodos normalizados.

Finalmente, se estudiaron mediante microscopía electrónica de barrido a $-190{ }^{\circ} \mathrm{C}$ (Crio-SEM), muestras representativas de los materiales finales obtenidos a partir del tratamiento de diferentes residuos. Las observaciones morfológicas se realizaron en un microscopio de barrido Zeiss modelo DSM 960. Las muestras, previamente metalizadas con oro, se observaron a $-190^{\circ} \mathrm{C}$ empleando la técnica de crio-scanning para evitar alteraciones en los compuestos orgánicos

\section{3.- RESULTADOS EXPERIMENTALES. DISCUSION}

La composición química de al arcilla, expresada en elementos mayoritarios corresponde a : $\mathrm{SiO}_{2}: 60.5 \% ; \mathrm{Al}_{2} \mathrm{O}_{3}: 2.4 \%$; $\mathrm{MgO}: 23.8 \%$; $\mathrm{CaO}: 0.5 \%$; $\mathrm{Fe}_{2} \mathrm{O}_{3}: 0.9 \%$; $\mathrm{Na}_{2} \mathrm{O}: 0.1 \%$; $\mathrm{K}_{2} \mathrm{O}: 0.5 \%$. La pérdida por calcinación a $1000^{\circ} \mathrm{C}$ es del $12.5 \%$ en peso.

Su composición mineralógica indica que el $85 \%$ de la arcilla corresponde a sepiolita y el resto está constituido por cuarzo, feldespato potásico e illita. La superficie específica presenta un valor de $320 \mathrm{~m}^{2} / \mathrm{g}$ y la porosidad total equivale a $0.83 \mathrm{~cm}^{3} . \mathrm{g}^{-1}$. Granulometricamente, el $98 \%$ del peso de la arcilla lo constituye partículas de tamaño inferior a $5 \mu \mathrm{m}$.

TABLA 2. CARACTERÍSTICAS FíSICAS Y QUíMICAS DE LOS MATERIALES FINALES

\begin{tabular}{|c|c|c|c|c|c|c|c|c|c|c|c|}
\hline $\begin{array}{c}\text { Mezclas } \\
\text { kg.arcilla/kg. } \\
\text { Residuo }\end{array}$ & $\begin{array}{c}\text { Descripción } \\
\text { física }\end{array}$ & $\begin{array}{c}\text { Densidad } \\
\left(\mathrm{g} / \mathrm{cm}^{3}\right)\end{array}$ & $\begin{array}{c}\text { Punto } \\
\text { Inflamación } \\
\left({ }^{\circ} \mathrm{C}\right)\end{array}$ & $\begin{array}{c}\text { PCS } \\
\text { (Kcal/kg) }\end{array}$ & $\begin{array}{c}\text { Humeda } \\
\text { d } \\
(\%)\end{array}$ & $\begin{array}{c}\text { Pérdida de } \\
\text { peso a } \\
105^{\circ} \mathrm{C}(\%)\end{array}$ & $\begin{array}{c}\text { Volátiles } \\
(\%)\end{array}$ & $\begin{array}{c}\text { Cloro } \\
\text { Total (\%) }\end{array}$ & $\begin{array}{l}\text { DQO } \\
(\mathrm{mg} / \mathrm{l})\end{array}$ & $\begin{array}{c}\text { EC50 } \\
\times 10^{4} \\
(\mathrm{mg} / \mathrm{l})\end{array}$ & $\begin{array}{c}\text { Metales } \\
(\mathrm{mg} / \mathrm{l})\end{array}$ \\
\hline \multicolumn{12}{|l|}{ Lodos Limpieza } \\
\hline 0.6 & sol. pul. & $0.57-0.75$ & $82-100$ & $500-3630$ & 4.4-31.5 & $15.9-33.8$ & $2.1-11.5$ & $0.13-2.83$ & De 7836 a & $>1.2$ & $<0.5$ \\
\hline 0.5 & sol. pul. & $0.47-0.55$ & $85-90$ & $500-3066$ & $4.8-26.4$ & $14.8-27.6$ & 9.9.-1.2 & $0.40-2.05$ & 11000 & $>1.2$ & $<0.5$ \\
\hline 0.4 & sol. pul. & $0.42-0.42$ & $90-100$ & $500-2690$ & $6.5-24.1$ & $11.8-25.9$ & $5.3-1.8$ & $0.22-1.84$ & & $>1.2$ & $<8$ \\
\hline \multicolumn{12}{|l|}{ Cosméticos } \\
\hline 0.5 & idem & $0.28-0.41$ & $72-92$ & $1318-2320$ & $8.1-24.1$ & $13.6-39.2$ & $5.5-15.1$ & $0.13-0.42$ & 3700 & -- & $<0.5$ \\
\hline 0.4 & idem & $0.26-0.35$ & $94-100$ & $1192-2108$ & $10.3-23.5$ & $11.6-31.3$ & 1.1-7.8 & $0.10-0.49$ & & 3.1 & $<0.5$ \\
\hline \multicolumn{12}{|l|}{ Resinas } \\
\hline 0.6 & sol. pul. & 0.80 & 83 & 5151 & 1.7 & 24.5 & 22.8 & 0.08 & $\ldots$ & $\ldots$ & $<0.5$ \\
\hline 0.5 & idem & 0.40 & 98 & 4217 & 5.2 & 17.1 & 11.9 & 0.07 & 3900 & 0.93 & $<0.5$ \\
\hline 0.4 & idem & 0.28 & 93 & 3339 & 8.8 & 14.0 & 5.2 & 0.06 & $\ldots$ & $\ldots$ & $<16$ \\
\hline \multicolumn{12}{|l|}{ Pinturas } \\
\hline 0.4 & idem & 0.52 & 95 & 2194 & 3.8 & 5.1 & 1.4 & 0.20 & 4500 & 0.58 & 29 \\
\hline \multicolumn{12}{|l|}{ Tensioactivos } \\
\hline 0.4 & sol.pul. & 0.79 & $>100$ & $<500$ & - & 37.1 & - & 12.43 & 579 & & 3.2 \\
\hline \multicolumn{12}{|l|}{ Muestra Media } \\
\hline 0.6 & sol.pul. & 0.24 & $>100$ & 1843 & 0.8 & 1.1 & 0.3 & 0.66 & 8605 & n.d & 3.8 \\
\hline 0.5 & idem & 0.39 & 92 & 2274 & 0.6 & 1.0 & 0.4 & 0.71 & 9000 & n.d & 4.4 \\
\hline 0.4 & idem & 0.55 & 80 & 2618 & 0.5 & 1.0 & 0.5 & 1.14 & 11560 & 0.95 & 4.9 \\
\hline
\end{tabular}

sol. pul.= sólido pulverulento 
Los residuos estudiados en este trabajo son líquidos, muy viscosos o sólidos pastosos difícilmente manejables. El punto de inflamación es, en la mayoría de los casos, inferior a $55^{\circ} \mathrm{C}$, en especial en los lodos, resinas y fondos de destilación, lo que les confiere un alto grado de peligrosidad por inflamabilidad.

El poder calorífico (PCS), es superior a $5000 \mathrm{Kcal} / \mathrm{Kg}$ en las resinas, en los fondos de destilación de pinturas y en gran parte de cosméticos y lodos de limpieza. Las pérdidas de peso por secado a $105^{\circ} \mathrm{C}$ corresponden a pérdidas de agua en el caso de tensioactivos y algunos cosméticos y a pérdidas por evaporación de disolventes, fundamentalmente, en el resto de los residuos.

Hay que señalar el carácter fuertemente ácido, $\mathrm{pH}<1$ de los tensioactivos, el elevado contenido en cloro tanto en tensioactivos como en algunos lodos de limpieza, y el elevado contenido en metales pesados en todos los grupos de residuos estudiados, especialmente en los fondos de destilación de pinturas y cosméticos.

En la Tabla 2 se recogen algunas de las características físico-químicas de los materiales finales obtenidos por el procedimiento descrito. Se trata de sólidos pulverulentos fácilmente manejables. Desde un punto de vista físico-químico, se observa que los materiales obtenidos presentan puntos de inflamación superiores a los del residuo de partida, en general, próximos a $100^{\circ} \mathrm{C}$ lo que significa un menor grado de inflamabilidad y peligrosidad. Los contenidos en cloro, azufre y volátiles disminuyen en todos los casos estudiados de manera proporcional al contenido de arcilla utilizada lo que indica un cierto efecto de dilución de los compuestos orgánicos en la mezcla.

En cuanto a las características químicas de los lixiviados, se observa que éstas dependen del residuo tratado, aunque en general, todos ellos presentan valores EC50 superiores a 3000 $\mathrm{mg} / \mathrm{l}$ y contenidos en metales muy bajos. En cambio, los valores de DQO son superiores a $2000 \mathrm{mg} / 1$ en todos los casos analizados excepto en los materiales obtenidos a partir de tensioactivos en cuyo caso se obtienen valores de DQO inferiores a $2000 \mathrm{mg} / 1$.

En cuanto a los resultados obtenidos en el tratamiento de la muestra media de residuos, se observa que los materiales finales presentan también puntos de inflamación muy superiores al inicial y proporcionales al contenido de arcilla en la mezcla. Así mismo, se produce una disminución importante de la humedad y de las pérdidas por secado a 105º así como del contenido en volátiles, lo que indica que los compuestos orgánicos libres han disminuido en relación con los existentes en el residuo inicial. Los contenidos de cloro disminuyen hasta un $70 \%$ en relación al contenido inicial y los contenidos en azufre se sitúan por debajo de $150 \mathrm{mg} / \mathrm{kg}$. Así mismo, los contenidos en metales disminuyen por debajo de $300 \mathrm{mg} / \mathrm{kg}$, lo que supone una disminución del $96 \%$ sobre los contenidos iniciales. En relación con los lixiviados, todos cumplen la normativa en cuanto al EC50 y contenido en metales, pero superan el límite establecido para la DQO.

En la Figura 1 se muestra un aspecto microscópico de la arcilla incial y de dos de las muestras de material inerte obtenidas con $0.6 \mathrm{~kg}$ arcilla $/ \mathrm{kg}$.inerte final. En la Figura 1(a) se observan cristales aciculares microfibrosos característicos de la sepiolita y en la Figura 1(b) aparecen aglomerados constituidos por sepiolita y materia orgánica. Se observa también (Figura 1(c)), que la arcilla conserva aún su estructura cristalina acicular característica incluso una vez que las moléculas orgánicas se han absorbido en ella.

En todas las partículas se observa una distribución relati-
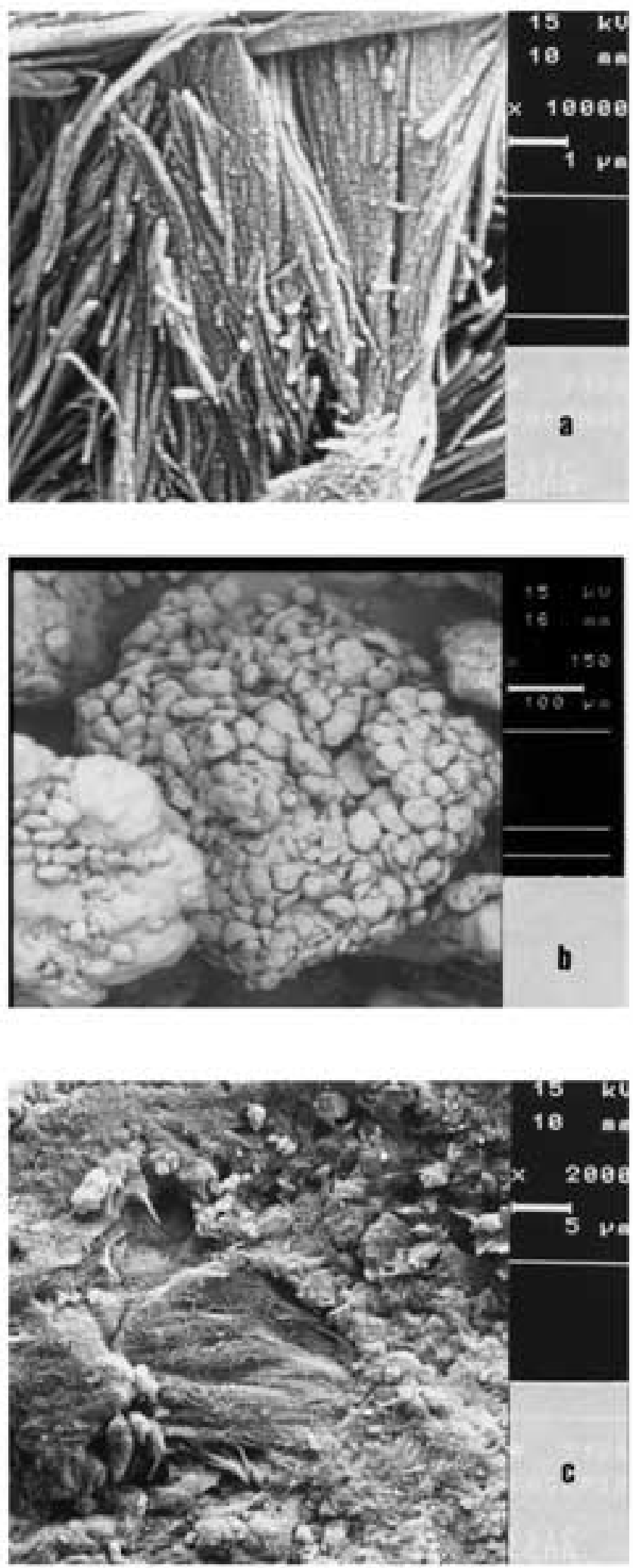

Figura 1.- Aspectos morfológicos observados mediante Crio-scanning a $-190^{\circ} \mathrm{C}$

a) Cristales aciculares y microfibrosos de sepiolita antes de su empleo en el proceso

b) Aglomerados de arcilla y compuestos orgánicos en un inerte que contiene $0.6 \mathrm{~kg}$. de arcilla $/ \mathrm{kg}$. de inerte

c) Cristales aciculares de sepiolita observados en un inerte. 
vamente homogénea de la materia orgánica, como se desprende de los resultados del análisis químico mediante energías dispersivas. Esto indica, que en las condiciones de trabajo señaladas y con las proporciones arcilla/residuo estudiadas, la totalidad del residuo interacciona con la arcilla, dando lugar a un material en el que los compuestos orgánicos se distribuyen de una manera homogénea.

Los materiales finales obtenidos presentan, en la mayoría de los casos, contenidos de halógenos totales iguales o inferiores al 2\%; contenido total en metales pesados muy inferior a 5000 ppm (límite máximo impuesto por algunas legislaciones) y en general, valores de PCS superiores a $2000 \mathrm{Kcal} / \mathrm{kg}$. Estos resultados hacen pensar que sería posible llevar a cabo la revalorización energética de los residuos, después de ser sometidos al proceso de solidificación/estabilización descrito. La revalorización podría llevarse a cabo en hornos rotativos para la fabricación de clinker (15). En estos hornos, se dan las condiciones adecuadas de temperatura (la llama alcanza los $2000^{\circ} \mathrm{C}$, alcanzándose en el interior del horno los $1450^{\circ} \mathrm{C}$ con temperaturas de los gases de $1200^{\circ} \mathrm{C}$ ); tiempo de residencia del residuo en el horno (de 5 a 6 segundos a $1200^{\circ} \mathrm{C}$ ); exceso de oxígeno tanto en la combustión como en la post-combustión; elevada turbulencia en el interior del horno; neutralización de los posibles gases formados durante la combustión (ácidos, óxidos de azufre y $\mathrm{HCl}$ ) mediante la cal activa que se encuentra en el propio horno y fijación de los posibles metales pesados en la estructura del clinker . Finalmente, este tratamiento no genera nuevos residuos sólidos o líquidos y permite extraer los valores caloríficos y energéticos contenidos en los materiales granulados y en consecuencia, disminuir el consumo de combustibles fósiles.

Por último, el proceso de estabilización desarrollado, permite adecuar las proporciones arcilla/residuo en función de las características físico-químicas de éste. En los casos estudiados, se ha comprobado que el contenido de residuo orgánico en las mezclas finales puede llegar a alcanzar la cantidad de $0.6 \mathrm{~kg}$ por $\mathrm{kg}$ de producto final siendo el resto arcilla. Algo similar sucede en el tratamiento de la muestra mezcla de residuos cuyo tratamiento puede realizarse utilizando $0.4 \mathrm{~kg}$ de arcilla por $\mathrm{kg}$ de producto final.

\section{4.- CONCLUSIONES}

El proceso desarrollado permite la solidificación/estabilización de residuos tóxicos y peligrosos de naturaleza orgánica. El proceso se basa en la absorción de las moléculas orgánicas en una arcilla de elevada superficie específica y estructura cristaloquímica adecuada. El resultado de este proceso, es un sólido granulado o semigranulado de punto de inflamación próximo a $100 \stackrel{\circ}{\circ}$.

Con la arcilla utilizada (sepiolita con superficie específica BET del orden de $300 \mathrm{~m}^{2} / \mathrm{g}$ ), se logra realizar mezclas, con buen comportamiento reológico, que pueden llegar a contener del orden de $0.6 \mathrm{~kg}$ de residuo orgánico por cada $\mathrm{kg}$ de materia inerte final. Los estudios realizados indican que sería posible introducir cantidades mayores de residuo, del orden de 0.8 $\mathrm{kg} / \mathrm{kg}$. inerte final aunque ello genera problemas de segregación y falta de homogeneidad en las mezclas obtenidas.

Como resultado del procedimiento desarrollado se llegan a obtener dos tipos de materiales finales. Por un lado, un sólido granulado, de baja humedad y punto de inflamación próximo a $100^{\circ} \mathrm{C}$, cuyo destino final puede ser un depósito de residuos. Por otro lado, un combustible de residuo (CDR) que podría ser revalorizado energéticamente en la producción del clinker, en cuyo caso, los materiales finales conseguidos aportarían elementos composicionales de interés, principalmente alúmina.

Utilizando una mezcla de todos los residuos estudiados, se obtienen materiales finales que podrían ser revalorizados energéticamente. En cambio, el tratamiento individualizado de cada uno de ellos permite obtener también materiales inertes que pueden ser depositados en vertederos adecuados.

Finalmente, y en el caso concreto de los residuos procedentes de la fabricación de pinturas, con altos contenidos en disolventes, estos se recuperan previamente al proceso de solidificación/estabilización con sepiolita.

\section{AGRADECIMIENTOS}

Este trabajo ha sido realizado con ayuda financiera de la Comunidad de Madrid (Dirección General de Investigación. Consejería de Educación y Cultura), Proyecto 06M/078/96.

Los autores agradecen a D. Miguel Ruiz, Dña. Pilar Rivera y Dña. Belén Morán de la empresa Cartera Ambiental S.A por el suministro y caracterización de los residuos estudiados.

Los autores agradecen la colaboración prestada por D. Fernando Pinto del Centro de Ciencias Medioambientales del CSIC en los estudios de microscopía, a D. Antonio Delgado y D. Carlos Pérez del Centro Nacional de Investigaciones Metalúrgicas (CSIC) por su ayuda en la realización de los ensayos experimentales.

\section{BIBLIOGRAFÍA}

1.- Z.Zhong, D.L.Sparks and N.C.Scrivner, "Sorption and Desorption of Quaternary Amine Cations on Clays". Environ.Sci.Technol. 27 1625-1631 (1993)

2.- M.Fernández Pérez. "Removal of Linuron from Water by Natural and Activated Bentonite" J.Chem.Technol Biotechnol 74 49-54 (1999).

3.- P.Fruhstorfer, R.J.Schneider, L.Weil and R. Niessner. "Factors Influencing the Adsorption of Atrazine on Montmorillonitic and Kaolinitic Clays" The Science of the Total Environment 138 317-328 (1993).

4.- E.González-Pradas, A.Valverde-García and M.Villafranca-Sánchez "Removal of Aromatic Amines from Aqueous Solutions by Activated Sepiolite" J.Chem.Technol. and Biotechnol 47 15-22 (1990).

5.- E.Srasra, F.Bergaya, H.Van Damme and N.K.Ariguib "Surface Properties of an Activated Bentonite-Decolorisation of Rape-Seed Oils" Applied Clay Science 4 411-421 (1989).

6.- R.Kondo, M.Daimon, C.Song and S.Jinawath. "Effect of lime on the hydration supersulfated slag cement". Am.Ceram.Soc.Bull., 59(8) 848-851 (1980).

7.- J J.Fritz. Process for rendering inert and/or immobilizing environmentally harmful susbtances. Patente WO 9101280 A1 910207.

8.- C.Cheeseman and E.Alcantara "Solidification of High Water Content Heavy Metal Sludges using Sepiolite and Smectite/Cement Mixes". Interim Report No.3 Imperial College of Science, Technologie and Medicine. London (England) 1996

9.- D.M. Montgomery, C.J.Sollars and R.Perry "Optimization of Cement-Based Stabilization/Solification of Organic Containing Industrial Wastes Using Organophilic Clays" Waste Manag.Research. 9 21-34 (1991).

10.- D.M. Montgomery, C.J. Sollars, T.S.Sheriff and R.Perry "OrganophilicClays for the Successful Stabilization/Solidification of Problematic Industrial Waste" Environ.Technol. Leters 9 1403-1412 (1988).

11.- T.A.Wolfe, T.Demirel and E.R.Baumann "Adsorption of Organic Pollutants on Montmorillonite Treated with Amines" J.Water Poll.Control Federation 58 68-76 (1986).

12.- F.A. López, A. López-Delgado, J. Núñez y M. Ruiz, "Procedimiento de estabilización e inertización de residuos orgánicos de carácter tóxico y peligroso". Patente española, P200002076, 11-Agosto-00 (2000)

13.- US EPA. "Bioassay for toxic and hazardous materials: training manual". Report 430/181026. US Environmental Protection Agency, California, 1981

14.- A.A.Bulich and D.L.Isemberg. "Use of the luminiscent bacterial system for the rapid assessment of aquatic toxicity". Instrument Society of American Trans., 20 29-33 (1981)

15.- M.Balbo; A.Francois; H.de Ladebas and F. Mosnier. "Cement kiln incineration. A viable waste management solution". pp. 123-127 In International Directory of Solid Waste Management. ISWA, Copenhagen, Denmark 1979. 